\title{
Relativistic Two-stream Instability
}

\author{
L. Samuelsson \\ Nordita, Roslagstullsbacken 23, 10691 Stockholm, Sweden \\ C. S. Lopez-Monsalvo, N. Andersson \\ School of Mathematics, University of Southampton \\ Southampton SO17 1BJ, UK \\ G. L. Comer \\ Department of Physics 8 Center for Fluids at All Scales, \\ Saint Louis University, St. Louis, MO, 63156-0907, USA
}

(Dated: July 28, 2021)

\begin{abstract}
We study the (local) propagation of plane waves in a relativistic, non-dissipative, two-fluid system, allowing for a relative velocity in the "background" configuration. The main aim is to analyze relativistic two-stream instability. This instability requires a relative flow - either across an interface or when two or more fluids interpenetrate - and can be triggered, for example, when onedimensional plane-waves appear to be left-moving with respect to one fluid, but right-moving with respect to another. The dispersion relation of the two-fluid system is studied for different two-fluid equations of state: (i) the "free" (where there is no direct coupling between the fluid densities), (ii) coupled, and (iii) entrained (where the fluid momenta are linear combinations of the velocities) cases are considered in a frame-independent fashion (eg. no restriction to the rest-frame of either fluid). As a by-product of our analysis we determine the necessary conditions for a two-fluid system to be causal and absolutely stable and establish a new constraint on the entrainment.
\end{abstract}

\section{INTRODUCTION}

Newtonian physics is replete with examples of multi-fluid systems, such as diffusion, ion flow, superfluid Helium, and plasma discharge from the Sun. In fact, large characteristic scattering times between different components is more the norm than the exception. This leads to physical situations where the various components can move independently of each other, be it across an interface or through interpenetration. In this context, even heat conduction in systems where all the matter flows together is a two-fluid problem, i.e. there is a heat flux in addition to the matter flux. Perhaps not as widely appreciated is that the relativistic regime has its own set of multi-fluid scenarios: neutrino streaming during supernovae, superfluid neutrons and superconducting protons in neutron stars, and heat flow in a cosmological setting, to name but a few.

A key issue is that relativistic fluids must be causal, meaning that sound speeds, say, must be less than that of light. For fluids, there are two entry points for causality: the microscopic where particle-particle interactions are tracked and the macroscopic where fluid elements (large enough to contain many particles, but small enough to be point-like with respect to the total system) are monitored. Presumably, a fully relativistic treatment at the microscopic level would lead to a set of fluid coefficients (describing the equation of state, dissipation, etc.) that would already behave appropriately at the macroscopic level. However, there is a practical problem: Equation of state determinations are notoriously difficult. This makes a general analysis of relativistic fluid dynamics prohibitive, if not impossible. Fortunately, one can make progress by imposing causality "from above" and absolute stability (i.e. real sound speeds) "from below" to constrain the fluid coefficients.

In this paper, we will do this by analyzing the local propagation of plane waves on a given (arbitrary) background spacetime. Compared to the standard single-fluid analysis, we have more fluid degrees of freedom and need to allow for relative flows between the various fluids. This is an essential requirement for two-stream instability. Such instabilities are known to exist for a variety of configurations. For shearing motion at an interface, it is an example of Kelvin-Helmholtz instability. However, as far as we are aware, generic two-stream instability has not been discussed previously in relativity.

The two-stream instability has been well-documented for plasmas (where it is known as the "Farley-Buneman" instability [1, 2], see [3] for a text-book discussion). It has also been suggested as the mechanism behind star formation when two galaxies (whose angular velocities are more or less anti-aligned) merge [4]. In the general relativistic context, Chandrasekhar, Friedman, and Schutz (CFS) [5, 6] have demonstrated that oscillation modes in rotating, perfect fluids can become two-stream unstable due to the emission of gravitational radiation. Here, the two "fluids" are the rotating mass, and the asymptotically flat spacetime in which the fluid is embedded. Most recently, a two-stream instability for superfluids has been proposed, with a natural extension to a mixture of superfluid neutrons and superconducting protons in neutron star cores [7, 8]. Very recent results suggest that this instability may act as 
a trigger mechanism for the enigmatic spin glitches that have been observed in a number of radio pulsars [9] (see also [8] for the first suggestion of a link between glitches and two-stream instability).

In what follows we will not restrict the discussion to any specific physical system. Consequently, the analysis will be somewhat abstract. This strategy can work because the two essential requirements for triggering twostream instability is a relative flow between two fluids and a generic interaction between them. This freedom to remain abstract illustrates the general robustness of the instability and its presence in a diverse collection of systems. Essentially, if the relative velocity is large enough that a wave moves in one direction with respect to the rest-frame of one fluid, yet the opposite direction with respect to the other fluid's rest-frame, then the energy of the wave will be "negative" in one of the rest-frames and therefore unbounded from below. Our main aim is to show that a causal and absolutely stable system of two relativistic fluids can undergo two-stream instability for a range of relative speeds and equation of state parameter values.

The presentation of the results is organized as follows: Section \ recalls the multi-fluid formalism, and sets the stage for a plane-wave analysis of the system. Section III considers sound waves for a single fluid. The results are not new, but they help establish basic techniques that carry over to the more complicated two-fluid calculations discussed in Section IV The following sub-sections consider three variations on the two-fluid equation of state: (i) the "free" (where there is no direct coupling between the fluid densities), (ii) coupled, and (iii) entrained cases. Finally, in Section $\mathrm{V}$, we make our concluding remarks. Spacetime indices are denoted by the first letters of the roman alphabet $(a, b, c)$, constituent indices by the last $(\mathrm{x}, \mathrm{y}, \mathrm{z})$, and we adopt "MTW" (Misner, Thorne, and Wheeler [10]) conventions for the metric signature.

\section{THE MULTI-FLUID FORMALISM}

We will use the approach to multi-fluid systems that was developed originally by Carter [11] (see [12] for a recent review). In this description, the main variables are the various fluxes (for particles and/or entropy), to be denoted $n_{\mathrm{x}}^{a}$, and the equations of motion follow from a suitably defined "master" function (i.e. Lagrangian or equation of state) $\Lambda$. In the single fluid case, $-\Lambda$ is equal to the rest frame energy density $\rho$. We have here introduced the convention of attaching a constituent index $\mathrm{x}$ to each variable. This index is redundant for a single fluid, but necessary when there are multiple fluids. The master function varies only with the fluxes. If the fluids are locally isotropic (i.e. no preferred direction), as they should be in the absence of anything else (such as an elastic solid), it is clear that $\Lambda$ must be a function of only the various scalars that can be formed from inner products of the fluxes.

We will focus on the case of two fluids (see 12] for a complete description), even though most of the equations in the general discussion will carry enough constituent indices ( $\mathrm{x}, \mathrm{y}$, etc.) to be valid for any number of fluids. In the case of two components, the master function depends on two distinct particle fluxes $n_{\mathrm{x}}^{a}$ and $n_{\mathrm{y}}^{a}$ and has the functional dependence [23]

$$
\Lambda=\Lambda\left(n_{\mathrm{x}}^{2}, n_{\mathrm{y}}^{2}, n_{\mathrm{xy}}^{2}\right),
$$

where $n_{\mathrm{xy}}^{2}=-g_{a b} n_{\mathrm{x}}^{a} n_{\mathrm{y}}^{b}$. Note that constituent indices are not summed over when repeated. As a matter of convenience repeated indices are written only once; that is, we write $n_{\mathrm{x}}^{2}$ (which is the squared particle number density of the $\mathrm{x}^{\text {th }}$-fluid) for $n_{\mathrm{xx}}^{2}$ and so on.

The equations of motion become most transparent when expressed in terms of the momentum $\mu_{a}^{\mathrm{x}}$ which is canonically conjugate to $n_{\mathrm{x}}^{a}$ :

$$
\mu_{a}^{\mathrm{x}}=\mathcal{B}^{\mathrm{x}} n_{a}^{\mathrm{x}}+\mathcal{A}^{\mathrm{xy}} n_{a}^{\mathrm{y}}
$$

where

$$
\mathcal{B}^{\mathrm{x}} \equiv-2 \frac{\partial \Lambda}{\partial n_{\mathrm{x}}^{2}} \quad, \quad \mathcal{A}^{\mathrm{xy}} \equiv-\frac{\partial \Lambda}{\partial n_{\mathrm{xy}}^{2}} .
$$

Note that we have simplified the notation by not indicating explicitly which variables are fixed when partial derivatives are taken. (The functional dependence of the master function is clear from (11).) From (2) we see that the momentum $\mu_{a}^{\mathrm{x}}$ is not simply proportional to its canonical conjugate $n_{\mathrm{x}}^{a}$, but is rather a linear combination of all the fluxes. This is a result of the so-called entrainment effect (see 13] for an example in superfluid Helium mixtures, 14, 15, 16] for relativistic, nuclear matter, or [17] for a treatment of entropy/matter entrainment and its importance for heat flow).

It is convenient at this point to introduce a shorthand notation for derivatives of these coefficients; namely,

$$
\mathcal{C}_{c c}^{2} \equiv \frac{1}{\mathcal{B}^{\mathrm{x}} \mathcal{B}^{\mathrm{y}}}\left(2 n_{\mathrm{x}} n_{\mathrm{y}} \frac{\partial \mathcal{B}^{\mathrm{x}}}{\partial n_{\mathrm{y}}^{2}}\right),
$$




$$
\begin{aligned}
\mathcal{B}_{, \mathrm{xy}}^{\mathrm{x}} & \equiv n_{\mathrm{x}} n_{\mathrm{y}} \frac{\partial \mathcal{B}^{\mathrm{x}}}{\partial n_{\mathrm{xy}}^{2}}, \\
\mathcal{A}_{, \mathrm{xy}}^{\mathrm{xy}} & \equiv n_{\mathrm{x}} n_{\mathrm{y}} \frac{\partial \mathcal{A}^{\mathrm{xy}}}{\partial n_{\mathrm{xy}}^{2}} .
\end{aligned}
$$

For the same reason we define the "speed-of-sound" $c_{\mathrm{x}}^{2}$ of the $\mathrm{x}^{\text {th }}$-fluid as

$$
c_{\mathrm{x}}^{2}=\frac{\partial \log \mathcal{B}^{\mathrm{x}}}{\partial \log n_{\mathrm{x}}}+1
$$

Finally, we introduce the "perp" operator

$$
\perp_{a}^{\mathrm{x} b}=\delta_{a}^{b}+u_{a}^{\mathrm{x}} u_{\mathrm{x}}^{b} \quad, \quad \perp_{a}^{\mathrm{x} b} u_{\mathrm{x}}^{a}=0,
$$

which can be used to construct, say, vectors that are orthogonal to $u_{\mathrm{x}}^{a}$.

We have chosen the fluxes $n_{\mathrm{x}}^{a}$ as our primary fields. However, there is no reason why the momenta $\mu_{a}^{\mathrm{x}}$ could not be similarly adopted 24]. This implies that the mapping from one set of fields to the other must have an inverse. That is, we see from (2) that

$$
\left[\begin{array}{l}
\mu_{a}^{\mathrm{x}} \\
\mu_{a}^{\mathrm{y}}
\end{array}\right]=\left[\begin{array}{cc}
\mathcal{B}^{\mathrm{x}} & \mathcal{A}^{\mathrm{xy}} \\
\mathcal{A}^{\mathrm{xy}} & \mathcal{B}^{\mathrm{y}}
\end{array}\right]\left[\begin{array}{l}
n_{a}^{\mathrm{x}} \\
n_{a}^{\mathrm{y}}
\end{array}\right]
$$

and thus $\mathcal{B}^{\mathrm{x}} \mathcal{B}^{\mathrm{y}}-\left(\mathcal{A}^{\mathrm{xy}}\right)^{2} \neq 0$. This will be a useful constraint later, when we discuss the impact of entrainment on sound modes.

For the purely variational case (i.e. no dissipation, imposed constraints, etc.), the individual constituents are conserved [12, 18], so that we have for each component

$$
\nabla_{a} n_{\mathrm{x}}^{a}=0 .
$$

The remaining equations of motion take the form

$$
n_{\mathrm{x}}^{a} \omega_{a b}^{\mathrm{x}}=0
$$

where the vorticity tensor $\omega_{a b}^{\mathrm{x}}$ is given by

$$
\omega_{a b}^{\mathrm{x}} \equiv 2 \nabla_{[a} \mu_{b]}^{\mathrm{x}} .
$$

As discussed, eg., in [12], in the single-fluid case these equations contain the same information as the standard set obtained from the vanishing of the covariant divergence of the stress-energy-momentum tensor. Eq. (9) illustrates the geometrical significance of the Euler equation as an integrability condition on the vorticity; i.e. that the particle flux nowhere pierces the surfaces defined by the two-form $\omega_{a b}^{\mathrm{x}}$.

Below we will be analyzing plane-wave propagation on backgrounds such that $\omega_{a b}^{\mathrm{x}}=0$, the various background quantities are taken to be constant, and there is a relative flow between the fluids. This implies a linearization of the equations of motion; i.e.

$$
\nabla_{a} \delta n_{\mathrm{x}}^{a}=0 \quad, \quad n_{\mathrm{x}}^{a} \nabla_{[a} \delta \mu_{b]}^{\mathrm{x}}=0 .
$$

Because there are several fluids, the variation $\delta \mu_{a}^{\mathrm{x}}$ is significantly more complicated than, say, in the case of the standard perfect fluid. In addition to individual bulk effects for each fluid, there can also be cross-constituent effects due to coupling between the fluids. We also have to consider the entrainment.

Following [12], we can isolate the various effects in the variation and write

$$
\delta \mu_{a}^{\mathrm{x}}=\left(\mathcal{B}_{a b}^{\mathrm{x}}+\mathcal{A}_{a b}^{\mathrm{x}}\right) \delta n_{\mathrm{x}}^{b}+\left(\mathcal{X}_{a b}^{\mathrm{xy}}+\mathcal{A}_{a b}^{\mathrm{xy}}\right) \delta n_{\mathrm{y}}^{b},
$$

where the bulk effects are captured by

$$
\mathcal{B}_{a b}^{\mathrm{x}}=\mathcal{B}^{\mathrm{x}}\left(\perp_{a b}^{\mathrm{x}}-c_{\mathrm{x}}^{2} u_{a}^{\mathrm{x}} u_{b}^{\mathrm{x}}\right)
$$

the cross-constituent coupling through

$$
\mathcal{X}_{a b}^{\mathrm{xy}}=-\mathcal{C}_{c c} \sqrt{\mathcal{B}^{\mathrm{x}} \mathcal{B}^{\mathrm{y}}} u_{a}^{\mathrm{x}} u_{b}^{\mathrm{y}}
$$


and the entrainment via the terms

$$
\begin{aligned}
& \mathcal{A}_{a b}^{\mathrm{x}}=-\left[\mathcal{B}_{, \mathrm{xy}}^{\mathrm{x}}\left(u_{a}^{\mathrm{x}} u_{b}^{\mathrm{y}}+u_{b}^{\mathrm{x}} u_{a}^{\mathrm{y}}\right)+\frac{n_{\mathrm{y}}}{n_{\mathrm{x}}} \mathcal{A}_{, \mathrm{xy}}^{\mathrm{xy}} u_{a}^{\mathrm{y}} u_{b}^{\mathrm{y}}\right], \\
& \mathcal{A}_{a b}^{\mathrm{xy}}=\mathcal{A}^{\mathrm{xy}} \perp_{a b}^{\mathrm{x}}-\left[\left(\mathcal{A}^{\mathrm{xy}}+\frac{n_{\mathrm{x}}}{n_{\mathrm{y}}} \mathcal{B}_{, \mathrm{xy}}^{\mathrm{x}}\right) u_{a}^{\mathrm{x}} u_{b}^{\mathrm{x}}+\frac{n_{\mathrm{y}}}{n_{\mathrm{x}}} \mathcal{B}_{, \mathrm{xy}}^{\mathrm{y}} u_{a}^{\mathrm{y}} u_{b}^{\mathrm{y}}+\mathcal{A}_{, \mathrm{xy}}^{\mathrm{xy}} u_{a}^{\mathrm{y}} u_{b}^{\mathrm{x}}\right] .
\end{aligned}
$$

In these expressions the flux $n_{\mathrm{x}}^{a}$ has been decomposed as $n_{\mathrm{x}}^{a}=n_{\mathrm{x}} u_{\mathrm{x}}^{a}$, where $u_{\mathrm{x}}^{a}$ is the unit $\left(u_{a}^{\mathrm{x}} u_{\mathrm{x}}^{a}=-1\right)$ four-velocity of the $\mathrm{x}$-fluid elements.

\section{SINGLE-FLUID SOUND WAVES}

To set the stage for the general analysis it is useful to first consider the nature of sound waves in a single, perfect fluid. To do this, we perform a local analysis of linear perturbations of the fluid (keeping the metric fixed) on a generic background. In particular, plane-wave propagation corresponds to the Ansatz

$$
\delta n_{\mathrm{x}}^{a}=A_{\mathrm{x}}^{a} \exp \left(i k_{b} x^{b}\right)
$$

where the amplitude $A_{\mathrm{x}}^{a}$ and wave four-vector $k^{a}$ have vanishing covariant derivatives. Recall that we assume all unperturbed quantities to be similarly constant. In particular, the background vorticity simply vanishes. From (12) above we have

$$
\delta \mu_{a}^{\mathrm{x}}=\mathcal{B}_{a b}^{\mathrm{x}} \delta n_{\mathrm{x}}^{b} .
$$

Note that we have continued to use a constituent index, even though we are dealing with a single fluid. This allows for some economy of presentation since many of the formulas will apply later, except that the index x will then range over two fluids. Of course, waves in a system are such that the constant wave vector $k_{a}$ is the same for all the fluids. Hence, it does not carry a constituent index.

For convenience we will work in the material frame associated with the fluid. This means that $k_{a}$ and $A_{\mathrm{x}}^{a}$ will each be written as two pieces by utilizing the "perp" operator introduced in (6):

$$
k_{a}=k_{\mathrm{x}}\left(\sigma_{\mathrm{x}} u_{a}^{\mathrm{x}}+\hat{k}_{a}^{\mathrm{x}}\right),
$$

where $\sigma_{\mathrm{x}}$ and the wave vector $k_{a}^{\mathrm{x}}\left(\right.$ with magnitude $\left.k_{\mathrm{x}}\right)$ are

$$
k_{\mathrm{x}} \sigma_{\mathrm{x}}=-k_{a} u_{\mathrm{x}}^{a} \quad, \quad k_{a}^{\mathrm{x}}=\perp_{\mathrm{x} a}^{b} k_{b} \equiv k_{\mathrm{x}} \hat{k}_{a}^{\mathrm{x}} .
$$

Similarly, we can decompose the wave amplitude as

$$
A_{\mathrm{x}}^{a}=A_{\|}^{\mathrm{x}} u_{\mathrm{x}}^{a}+A_{\mathrm{x} \perp}^{a}
$$

where

$$
A_{\|}^{\mathrm{x}}=-u_{a}^{\mathrm{x}} A_{\mathrm{x}}^{a} \quad, \quad A_{\mathrm{x} \perp}^{a}=\perp_{\mathrm{x} b}^{a} A_{\mathrm{x}}^{b} .
$$

Note that $\sigma_{\mathrm{x}}$ and $\hat{k}_{\mathrm{x}}^{a}$ are measured by an observer moving with the fluid. It will be obvious from the dispersion relation constructed below that $\sigma_{\mathrm{x}}$ measures the phase velocity of the waves as seen in the fluid frame. Furthermore, it is easy to show that evaluating $\sigma_{\mathrm{x}}$ in a frame moving relative to the fluid leads to the standard Lorentz transformation of velocities.

With these preliminaries, the perturbation equations (11) reduce to

$$
\begin{aligned}
& 0=k_{a} A_{\mathrm{x}}^{a}, \\
& 0=n_{\mathrm{x}}^{a} k_{[a} \mathcal{B}_{b] c}^{\mathrm{x}} A_{\mathrm{x}}^{c} .
\end{aligned}
$$

The first of these relations shows that the waves are transverse in the spacetime sense. The dispersion relation can be easily obtained by contracting the second equation with $k^{b}$. (In the more complicated two-fluid analysis below, we will in general have to consider the vanishing of a $4 \times 4$ determinant.) Assuming that $n_{c}^{\mathrm{x}} A_{\mathrm{x}}^{c} \neq 0$, and after several steps of algebra, the dispersion relation reduces to

$$
\sigma_{\mathrm{x}}^{2}-c_{\mathrm{x}}^{2}=0 .
$$


In our homogeneous plane-wave setting it is clear that the group and phase velocities coincide so that we can introduce the speed of sound in the standard way as $c_{\mathrm{x}}^{2}=\sigma_{\mathrm{x}}^{2}$.

To see that this is equivalent to the usual single-fluid result (as in, say, [19]), it will suffice to introduce the pressure and recall that $\rho=-\Lambda$ in the single fluid case. From the definition above for $\mathcal{B}^{\mathrm{x}}$, cf. (3), we see that

$$
\mathrm{d} \rho=\mathcal{B}^{\mathrm{x}} n_{\mathrm{x}} \mathrm{d} n_{\mathrm{x}} .
$$

Moreover, the pressure $p$ is defined by the standard thermodynamic relation, such that

$$
p=-\rho+n_{\mathrm{x}} \frac{\mathrm{d} \rho}{\mathrm{d} n_{\mathrm{x}}}=-\rho+\mathcal{B}^{\mathrm{x}} n_{\mathrm{x}}^{2} .
$$

This implies

$$
\mathrm{d} p=\left(1+\frac{\partial \log \mathcal{B}^{\mathrm{x}}}{\partial \log n_{\mathrm{x}}}\right) \mathrm{d} \rho,
$$

and we have

$$
\frac{\mathrm{d} p}{\mathrm{~d} \rho}=1+\frac{\partial \log \mathcal{B}^{\mathrm{x}}}{\partial \log n_{\mathrm{x}}}=c_{\mathrm{x}}^{2},
$$

where $\mathrm{d} p / \mathrm{d} \rho$ is the usual form of the sound speed squared.

In order to pave the way for the more complicated multi-fluid case to be discussed below it is useful to examine the properties of the various vectors we have introduced. Starting with the wave vector $k^{a}$ we see that

$$
k_{a} k^{a}=k_{\mathrm{x}}^{2}\left(1-c_{\mathrm{x}}^{2}\right) .
$$

Thus, for causal wave propagation $\left(c_{\mathrm{x}}^{2} \leq 1\right), k^{a}$ is spacelike. For the wave amplitude we find that, when the force equation (24) is evaluated in terms of the solution to the dispersion relation,

$$
A_{\mathrm{x} \perp}^{a}=\sigma_{\mathrm{x}} A_{\|}^{\mathrm{x}} \hat{k}_{\mathrm{x}}^{a} .
$$

The waves are therefore longitudinal in the normal, three-dimensional sense. On the other hand, the transverse nature (23) of the waves in the four-dimensional sense implies that

$$
A_{\mathrm{x}}^{2}=A_{\mathrm{x} \perp}^{2}\left(1-c_{\mathrm{x}}^{-2}\right)
$$

so that $A_{\mathrm{x}}^{a}$ is timelike (and we can choose it to be future pointing) for causal waves. Note that since the flux $n_{\mathrm{x}}^{a}$ is also timelike this implies that $n_{\mathrm{x}}^{a} A_{a}^{\mathrm{x}}<0$ and thus the degenerate case of (25) is ruled out by causality.

Before we conclude this section, it is useful to consider what constraints (5) imposes on the equation of state. First of all, the causality requirement leads immediately to

$$
\frac{\partial \log \mathcal{B}^{\mathrm{x}}}{\partial \log n_{\mathrm{x}}}+1 \leq 1
$$

In addition, we must have $c_{\mathrm{x}}^{2} \geq 0$ in order to avoid absolute instabilities (complex wave speeds). This implies

$$
\frac{\partial \log \mathcal{B}^{\mathrm{x}}}{\partial \log n_{\mathrm{x}}}+1 \geq 0
$$

Combining the two results we see that we must have

$$
-1 \leq \frac{\partial \log \mathcal{B}^{\mathrm{x}}}{\partial \log n_{\mathrm{x}}} \leq 0
$$

In the next section we will extend this type of analysis to the two-fluid model. 


\section{SOUND WAVES FOR THE GENERAL TWO-FLUID SYSTEM}

We want to work out the dispersion relation for wave propagation in a two-fluid system, using (17) as the starting point. An important addition to the problem of plane waves is a new "parameter", the relative flow between the two fluids. We will represent this flow by the relative velocity $v_{\mathrm{xy}}^{a}$ of the $\mathrm{y}^{\text {th }}$-fluid with respect to the frame of the $\mathrm{x}^{\text {th }}$-fluid:

$$
\gamma_{\mathrm{xy}} v_{\mathrm{xy}}^{a}=\perp_{b}^{\mathrm{x} a} u_{\mathrm{y}}^{b}
$$

where $v_{\mathrm{xy}}$ represents the magnitude of the relative flow and

$$
\gamma_{\mathrm{xy}}=\gamma_{\mathrm{yx}}=-u_{\mathrm{x}}^{c} u_{c}^{\mathrm{y}}=\frac{1}{\sqrt{1-v_{\mathrm{xy}}^{2}}} .
$$

This leads to

$$
u_{\mathrm{y}}^{a}=\gamma_{\mathrm{xy}}\left(u_{\mathrm{x}}^{a}+v_{\mathrm{xy}}^{a}\right) .
$$

The mode speed $\sigma_{\mathrm{x}}$ and wave (three-) vector $k_{a}^{\mathrm{x}}$ can be defined as before. The insertion of $\gamma_{\mathrm{xy}}$ into (36) makes the $\mathrm{x}^{\text {th }}$-fluid's proper time the standard for setting velocities. Since the dispersion relation below is a scalar equation, we will have in several places the inner product $\hat{v}_{\mathrm{xy}}^{a} \hat{k}_{a}^{\mathrm{x}}\left(\right.$ where $\left.\hat{v}_{\mathrm{xy}}^{a}=v_{\mathrm{xy}}^{a} / v_{\mathrm{xy}}\right)$. It is useful to write this in terms of the angle $\theta_{\mathrm{xy}}$ between the two vectors:

$$
\hat{v}_{\mathrm{xy}}^{a} \hat{k}_{a}^{\mathrm{x}}=\cos \theta_{\mathrm{xy}} .
$$

An important subtlety must be recognized, however: The three quantities $\sigma_{\mathrm{x}}, k_{a}^{\mathrm{x}}$, and $v_{\mathrm{xy}}^{a}$ are what would be measured by an observer flowing with the $\mathrm{x}^{\text {th }}$-fluid. We could have equally as well chosen the material frame attached to the other fluid (or some other observer). As one might expect, there are well-defined transformations between the two descriptions (which will be needed later). The relative flow $v_{\mathrm{yx}}^{a}$ of the $\mathrm{x}^{\text {th }}$-fluid with respect to the $\mathrm{y}^{\text {th }}$-fluid frame is related to $v_{\mathrm{xy}}^{a}$ via

$$
v_{\mathrm{yx}}^{a}=-\gamma_{\mathrm{xy}}\left(v_{\mathrm{xy}}^{2} u_{\mathrm{x}}^{a}+v_{\mathrm{xy}}^{a}\right),
$$

where we have used $v_{\mathrm{yx}}=v_{\mathrm{xy}}$. It is also useful to note that

$$
\begin{aligned}
& u_{\mathrm{x}}^{a}=-v_{\mathrm{xy}}^{-2}\left(v_{\mathrm{xy}}^{a}+\gamma_{\mathrm{xy}}^{-1} v_{\mathrm{yx}}^{a}\right), \\
& u_{\mathrm{y}}^{a}=-v_{\mathrm{xy}}^{-2}\left(v_{\mathrm{yx}}^{a}+\gamma_{\mathrm{xy}}^{-1} v_{\mathrm{xy}}^{a}\right) .
\end{aligned}
$$

Because $k_{a}$ is not attached to either fluid frame, we must have

$$
k_{a}=k_{\mathrm{y}}\left(\sigma_{\mathrm{y}} u_{a}^{\mathrm{y}}+\hat{k}_{a}^{\mathrm{y}}\right)=k_{\mathrm{x}}\left(\sigma_{\mathrm{x}} u_{a}^{\mathrm{x}}+\hat{k}_{a}^{\mathrm{x}}\right) .
$$

By contracting each four-velocity in (41) with the wave-vector $k_{a}$, we obtain a matrix equation for $\left[k_{\mathrm{x}} k_{\mathrm{y}}\right]^{\mathrm{T}}$; namely,

$$
\left[\begin{array}{cc}
v_{\mathrm{xy}} \sigma_{\mathrm{x}}-\cos \theta_{\mathrm{xy}} & -\gamma_{\mathrm{xy}}^{-1} \cos \theta_{\mathrm{yx}} \\
-\gamma_{\mathrm{xy}}^{-1} \cos \theta_{\mathrm{xy}} & v_{\mathrm{xy}} \sigma_{\mathrm{y}}-\cos \theta_{\mathrm{yx}}
\end{array}\right]\left[\begin{array}{l}
k_{\mathrm{x}} \\
k_{\mathrm{y}}
\end{array}\right]=\left[\begin{array}{l}
0 \\
0
\end{array}\right]
$$

Obviously the determinant of the $2 \times 2$ matrix must vanish. This leads to

$$
\sigma_{\mathrm{y}}=\cos \theta_{\mathrm{yx}} \frac{\sigma_{\mathrm{x}}-v_{\mathrm{xy}} \cos \theta_{\mathrm{xy}}}{v_{\mathrm{xy}} \sigma_{\mathrm{x}}-\cos \theta_{\mathrm{xy}}} .
$$

It is not difficult to show that if $\sigma_{\mathrm{x}}^{2} \leq 1$ then $\sigma_{\mathrm{y}}^{2} \leq 1$. This is natural given that causality is a frame-independent requirement.

Meanwhile, the equation of flux conservation is the same as (23) (except $\mathrm{x}$ ranges over two values). The conservation of vorticity equations become

$$
\begin{aligned}
& 0=K_{a b}^{\mathrm{x}} A_{\mathrm{x}}^{b}+K_{a b}^{\mathrm{xy}} A_{\mathrm{y}}^{b}, \\
& 0=K_{a b}^{\mathrm{y}} A_{\mathrm{y}}^{b}+K_{a b}^{\mathrm{yx}} A_{\mathrm{x}}^{b},
\end{aligned}
$$


where the "dispersion" tensors are

$$
\begin{aligned}
& K_{a b}^{\mathrm{x}}=n_{\mathrm{x}}^{c}\left(k_{[c} \mathcal{B}_{a] b}^{\mathrm{x}}+k_{[c} \mathcal{A}_{a] b}^{\mathrm{x}}\right), \\
& K_{a b}^{\mathrm{xy}}=n_{\mathrm{x}}^{c}\left(k_{[c} \mathcal{X}_{a] b}^{\mathrm{xy}}+k_{[c} \mathcal{A}_{a] b}^{\mathrm{xy}}\right) .
\end{aligned}
$$

Note that $K_{a b}^{\mathrm{y}}$ and $K_{a b}^{\mathrm{yx}}$ are obtained via the interchange of $\mathrm{x} \leftrightarrow \mathrm{y}$ in equation (46).

In order to solve (45), we obviously need the four inverses

$$
\tilde{K}_{\mathrm{x}}^{a c} K_{c b}^{\mathrm{x}}=\delta^{a}{ }_{c} \quad, \quad \tilde{K}_{\mathrm{yx}}^{a c} K_{c b}^{\mathrm{xy}}=\delta^{a}{ }_{c},
$$

to exist-i.e. the determinants of $K_{a b}^{\mathrm{x}}$ and $K_{a b}^{\mathrm{xy}}$ do not vanish-so that we can write

$$
0=\left(\tilde{K}_{\mathrm{y}}^{a c} K_{c b}^{\mathrm{yx}}-\tilde{K}_{\mathrm{yx}}^{a c} K_{c b}^{\mathrm{x}}\right) A_{\mathrm{x}}^{b} \equiv \mathcal{M}_{a b} A_{\mathrm{x}}^{b} .
$$

The only way to get a non-trivial solution is to have a $k_{a}$ such that

$$
\epsilon^{a_{1} a_{2} a_{3} a_{4}} \epsilon^{b_{1} b_{2} b_{3} b_{4}} \mathcal{M}_{a_{1} b_{1}} \mathcal{M}_{a_{2} b_{2}} \mathcal{M}_{a_{3} b_{3}} \mathcal{M}_{a_{4} b_{4}}=0 .
$$

Written out in full (49) is a quite busy expression. This should come as no surprise since the two-fluid problem is significantly more complicated than a single fluid having bulk contributions coming from $\mathcal{B}_{a b}^{\mathrm{x}}$, and the cross-coupling from $\mathcal{X}_{a b}^{\mathrm{xy}}$ (in the case of two, co-moving constituents [12]). For two-fluid systems, the two constituents move independently and there are the additional contributions $\mathcal{A}_{a b}^{\mathrm{x}}$ and $\mathcal{A}_{a b}^{\mathrm{xy}}$ coming from entrainment.

In order to simplify the problem, it is convenient to isolate further the different contributions that appear in the dispersion matrices. The bulk contribution in $K_{a b}^{\mathrm{x}}$ can be reduced to

$$
\begin{aligned}
b_{a b}^{\mathrm{x}} & =n_{\mathrm{x}}^{c} k_{[c} \mathcal{B}_{a] b}^{\mathrm{x}} \\
& =-\frac{1}{2} \mathcal{B}^{\mathrm{x}} n_{\mathrm{x}}\left(k_{\mathrm{x}} \sigma_{\mathrm{x}} \perp_{a b}^{\mathrm{x}}+c_{\mathrm{x}}^{2} k_{a}^{\mathrm{x}} u_{b}^{\mathrm{x}}\right),
\end{aligned}
$$

while its entrainment piece becomes

$$
\begin{aligned}
a_{a b}^{\mathrm{x}}= & n_{\mathrm{x}}^{c} k_{[c} \mathcal{A}_{a] b}^{\mathrm{x}} \\
= & \frac{1}{2} \gamma_{\mathrm{xy}} n_{\mathrm{x}}\left\{\mathcal{B}_{, \mathrm{xy}}^{\mathrm{x}}\left[\left(k_{\mathrm{x}} \sigma_{\mathrm{x}} v_{a}^{\mathrm{xy}}-2 k_{a}^{\mathrm{x}}\right) u_{b}^{\mathrm{x}}-k_{a}^{\mathrm{x}} v_{b}^{\mathrm{xy}}\right]\right. \\
& \left.+\gamma_{\mathrm{xy}} \frac{n_{\mathrm{y}}}{n_{\mathrm{x}}} \mathcal{A}_{, \mathrm{xy}}^{\mathrm{xy}}\left(k_{\mathrm{x}} \sigma_{\mathrm{x}} v_{a}^{\mathrm{xy}}-k_{a}^{\mathrm{x}}\right)\left(u_{b}^{\mathrm{x}}+v_{b}^{\mathrm{xy}}\right)\right\} .
\end{aligned}
$$

Meanwhile, the cross-coupling in $K_{a b}^{\mathrm{xy}}$ is simply given by

$$
\begin{aligned}
x_{a b}^{\mathrm{xy}} & =n_{\mathrm{x}}^{c} k_{[c} \mathcal{X}_{a] b}^{\mathrm{xy}} \\
& =-\frac{1}{2} \mathcal{C}_{c c} \gamma_{\mathrm{xy}} n_{\mathrm{x}} \sqrt{\mathcal{B}^{\mathrm{x}} \mathcal{B}^{\mathrm{y}}} k_{a}^{\mathrm{x}}\left(u_{b}^{\mathrm{x}}+v_{b}^{\mathrm{xy}}\right),
\end{aligned}
$$

but the entrainment has significantly more presence:

$$
\begin{aligned}
a_{a b}^{\mathrm{xy}}= & n_{\mathrm{x}}^{c} k_{[c} \mathcal{A}_{a] b}^{\mathrm{xy}} \\
= & \frac{n_{\mathrm{x}}}{2}\left\{-\mathcal{A}^{\mathrm{xy}} k_{\mathrm{x}} \sigma_{\mathrm{x}} \perp_{a b}^{\mathrm{x}}-\left[\mathcal{A}^{\mathrm{xy}}+\frac{n_{\mathrm{x}}}{n_{\mathrm{y}}} \mathcal{B}_{, \mathrm{xy}}^{\mathrm{x}}+\gamma_{\mathrm{xy}}\left(\gamma_{\mathrm{xy}} \frac{n_{\mathrm{y}}}{n_{\mathrm{x}}} \mathcal{B}_{, \mathrm{xy}}^{\mathrm{y}}+\mathcal{A}_{, \mathrm{xy}}^{\mathrm{xy}}\right)\right] k_{a}^{\mathrm{x}} u_{b}^{\mathrm{x}}\right. \\
& \left.+\gamma_{\mathrm{xy}} k_{\mathrm{x}} \sigma_{\mathrm{x}}\left(\gamma_{\mathrm{xy}} \frac{n_{\mathrm{y}}}{n_{\mathrm{x}}} \mathcal{B}_{, \mathrm{xy}}^{\mathrm{y}}+\mathcal{A}_{, \mathrm{xy}}^{\mathrm{xy}}\right) v_{a}^{\mathrm{xy}} u_{b}^{\mathrm{x}}\right\} .
\end{aligned}
$$

A multi-fluid system must have non-zero bulk properties (unless a fluid vanishes completely). The other terms can be absent, depending on the equation of state. In what follows we will systematically increase the complexity by considering in turn the different components of two-fluid physics. 


\section{A. Dispersion Relation: Free Case}

Let us first consider the case of two completely uncoupled fluids. Then we have only $b_{a b}^{\mathrm{x}}$ non-zero. In lieu of (49), it is easier to get the dispersion relation by contracting the free indices in (45) with $k_{a}$. This results in the simple $2 \times 2$ matrix problem

$$
\left[\begin{array}{cc}
\mathcal{B}^{\mathrm{x}}\left(\sigma_{\mathrm{x}}^{2}-c_{\mathrm{x}}^{2}\right) & 0 \\
0 & \mathcal{B}^{\mathrm{y}}\left(\sigma_{\mathrm{y}}^{2}-c_{\mathrm{y}}^{2}\right)
\end{array}\right]\left[\begin{array}{l}
u_{a}^{\mathrm{x}} A_{\mathrm{x}}^{a} \\
u_{a}^{\mathrm{y}} A_{\mathrm{y}}^{a}
\end{array}\right]=\left[\begin{array}{l}
0 \\
0
\end{array}\right]
$$

and the dispersion relation is simply

$$
\left(\sigma_{\mathrm{x}}^{2}-c_{\mathrm{x}}^{2}\right)\left(\sigma_{\mathrm{y}}^{2}-c_{\mathrm{y}}^{2}\right)=0 .
$$

By construction $\sigma_{\mathrm{x}}^{2}$ is the squared phase (three-) velocity as measured in the $\mathrm{x}^{\text {th }}$-fluid frame (similarly for $\sigma_{\mathrm{y}}^{2}$ ).

The outcome of this analysis is that the dispersion relation (55) allows four non-trivial solutions consisting of the roots of

$$
\sigma_{\mathrm{x}}^{2}=c_{\mathrm{x}}^{2}
$$

for any $\mathrm{x}$. These roots correspond to $\pm c_{\mathrm{x}}$ evaluated in the $\mathrm{x}^{\text {th }}$-fluid frame and are just Lorentz transformed if evaluated in another frame. Thus, as expected in the case of zero coupling between the fluids, the quantity $c_{\mathrm{x}}$ can be interpreted as the sound velocity of the $x^{\text {th }}$-fluid as measured in its own (background) rest frame. This is the obvious generalisation of the single fluid result. It follows that if $c_{\mathrm{x}}$ is subluminal in its own rest-frame it is so in all other frames as well. Also, since absolute instability should be evaluated at zero relative velocity it is clear that the constraint $0 \leq c_{\mathrm{x}}^{2} \leq 1$ remains as a condition for the master function.

The main conclusion from this discussion is that the constraints on the equation of state are easily generalized to the uncoupled two-fluid problem. We must thus require that the equation of state satisfy (35) for both fluids in order for the system to be absolutely stable and give rise to causal wave propagation. There are no dynamical instabilities present in this case. In what follows, we caution that the $c_{\mathrm{x}}$ can be understood as "sound" speeds only in this completely free case. When fluid couplings are operative, the phase velocities will no longer simply equal these free sound speeds. But in order to make progress, we will continue to impose (35) throughout.

\section{B. Dispersion Relation: Cross-constituent Coupling}

We now allow for $x_{a b}^{\mathrm{xy}}$ to be non-zero. From (52), we see that this will introduce $\mathcal{C}_{c c}$ in addition to $\mathcal{B}^{\mathrm{x}}$ and $c_{\mathrm{x}}^{2}$. Using the same contractions with $k_{a}$ as in the free case, we again get a $2 \times 2$ matrix problem; i.e.

$$
\left[\begin{array}{cc}
\mathcal{B}^{\mathrm{x}}\left(\sigma_{\mathrm{x}}^{2}-c_{\mathrm{x}}^{2}\right) & -\sqrt{\mathcal{B}^{\mathrm{x}} \mathcal{B}^{\mathrm{y}}} \mathcal{C}_{c c} \\
-\sqrt{\mathcal{B}^{\mathrm{x}} \mathcal{B}^{\mathrm{y}}} \mathcal{C}_{c c} & \mathcal{B}^{\mathrm{y}}\left(\sigma_{\mathrm{y}}^{2}-c_{\mathrm{y}}^{2}\right)
\end{array}\right]\left[\begin{array}{l}
u_{a}^{\mathrm{x}} A_{\mathrm{x}}^{a} \\
u_{a}^{\mathrm{y}} A_{\mathrm{y}}^{a}
\end{array}\right]=\left[\begin{array}{l}
0 \\
0
\end{array}\right] .
$$

The dispersion relation is now

$$
\left(\sigma_{\mathrm{x}}^{2}-c_{\mathrm{x}}^{2}\right)\left(\sigma_{\mathrm{y}}^{2}-c_{\mathrm{y}}^{2}\right)=\mathcal{C}_{c c}^{2} .
$$

In order for $\mathcal{C}_{c c}^{2}$ to be less than zero, the equation of state would have to allow either of the $\mathcal{B}^{\mathrm{x}}$ to be negative. For ordinary matter, or entropy, this is not generally the case. Hence, we do not consider this possibility here. We also need to point out that (44) must be used to get a dispersion relation solely in terms of $\sigma_{\mathrm{x}}$. Clearly, the cross-coupled case is more complicated than the free problem. However, it is also much more interesting and relevant. As we will soon see, the richer phenomenology allows for two-stream instability.

Addressing first the question of absolute stability we set $v_{\mathrm{xy}}=0$ to find

$$
\sigma_{\mathrm{x}}^{2}=\frac{1}{2}\left(c_{\mathrm{x}}^{2}+c_{\mathrm{y}}^{2} \pm \sqrt{\left(c_{\mathrm{x}}^{2}-c_{\mathrm{y}}^{2}\right)^{2}+4 \mathcal{C}_{c c}^{2}}\right) .
$$

In order to avoid complex $\sigma_{\mathrm{x}}^{2}$, the discriminant of (59) must be positive, which is evident for $\mathcal{C}_{c c}^{2} \geq 0$. Recall that absolute stability means $\sigma_{\mathrm{x}}^{2} \geq 0$. Clearly, it is sufficient to require that

$$
\left(c_{\mathrm{x}}^{2}+c_{\mathrm{y}}^{2}\right)^{2} \geq\left(c_{\mathrm{x}}^{2}-c_{\mathrm{y}}^{2}\right)^{2}+4 \mathcal{C}_{c c}^{2} .
$$


The second term of (59) will always be less than the first if

$$
c_{\mathrm{x}}^{2} c_{\mathrm{y}}^{2} \geq \mathcal{C}_{c c}^{2}
$$

In other words, since we expect to have $\mathcal{B}^{\mathrm{x}} \mathcal{B}^{\mathrm{y}}>0$, absolute stability constrains the equation of state to satisfy

$$
\frac{\partial \log \mathcal{B}^{\mathrm{x}}}{\partial \log n_{\mathrm{y}}} \frac{\partial \log \mathcal{B}^{\mathrm{y}}}{\partial \log n_{\mathrm{x}}} \leq\left(1+\frac{\partial \log \mathcal{B}^{\mathrm{x}}}{\partial \log n_{\mathrm{x}}}\right)\left(1+\frac{\partial \log \mathcal{B}^{\mathrm{y}}}{\partial \log n_{\mathrm{y}}}\right) .
$$

The causality constraint requires $\sigma_{\mathrm{x}}^{2} \leq 1$. For $\mathcal{C}_{c c}^{2} \geq 0$ and satisfying (61), we need only make the "+" solution in (59) causal, since the "-" solution is always smaller. We find that causality is ensured if

$$
\mathcal{C}_{c c}^{2} \leq\left(1-c_{\mathrm{x}}^{2}\right)\left(1-c_{\mathrm{y}}^{2}\right)
$$

which, in terms of the equation of state, translates into

$$
\frac{\partial \log \mathcal{B}^{\mathrm{x}}}{\partial \log n_{\mathrm{y}}} \frac{\partial \log \mathcal{B}^{\mathrm{y}}}{\partial \log n_{\mathrm{x}}} \leq \frac{\partial \log \mathcal{B}^{\mathrm{x}}}{\partial \log n_{\mathrm{x}}} \frac{\partial \log \mathcal{B}^{\mathrm{y}}}{\partial \log n_{\mathrm{y}}} .
$$

Note that both (61) and (63) restrict $\mathcal{C}_{c c}^{2}$ from above. In general, we can show that if $c_{\mathrm{x}}^{2}+c_{\mathrm{y}}^{2} \leq 1$ then any absolutely stable equation of state is also causal. Conversely, if $c_{\mathrm{x}}^{2}+c_{\mathrm{y}}^{2} \geq 1$, any causal equation of state is absolutely stable.

Given an equation of state that is causal and absolutely stable, we can now determine if a dynamical two-stream instability is present by solving the dispersion relation (58) for some relative flow (as parameterized by $v_{\mathrm{xy}}$ ). Writing down the general solution is not difficult, but it is instructive to first focus on the slow velocity limit. Assuming that $v_{\mathrm{xy}}$ and $\sigma_{\mathrm{x}}$ are both much smaller than unity (the speed of light) in (44), the dispersion relation (58) becomes

$$
\left(\sigma_{\mathrm{x}}^{2}-c_{\mathrm{x}}^{2}\right)\left[\left(\sigma_{\mathrm{x}}-v_{\mathrm{xy}} \cos \theta_{\mathrm{xy}}\right)^{2}-c_{\mathrm{y}}^{2}\right]=\mathcal{C}_{c c}^{2} .
$$

Introducing new variables

$$
x=\frac{\sigma_{\mathrm{x}}}{c_{\mathrm{y}}} \quad, \quad y=\frac{v_{\mathrm{xy}} \cos \theta_{\mathrm{xy}}}{c_{\mathrm{y}}} \quad, \quad b^{2}=\left(\frac{c_{\mathrm{x}}}{c_{\mathrm{y}}}\right)^{2} \quad, \quad a^{2}=\frac{\mathcal{C}_{c c}^{2}}{c_{\mathrm{y}}^{4}},
$$

we get

$$
\frac{x^{2}-b^{2}}{a^{2}}\left[(x-y)^{2}-1\right]=1 .
$$

As one might have expected, the problem is now identical to the Newtonian plane-wave problem discussed by Andersson, Comer, and Prix [8]. Hence, we can learn from their results. They demonstrate that a two-stream instability may operate above a critical relative flow. Their particular example corresponds to $a^{2}=0.0249$ and $b^{2}=0.0379$. For this case they find an instability in the range $0.6<y<1.5$. This means that the system becomes unstable for $c_{\mathrm{x}} y>0.6$. This flow is clearly sub-luminal as long as $c_{\mathrm{x}}<1$, but one may suspect that the linear approximation that we have used is not very accurate. Still, this is a useful first demonstration that the two-stream instability will operate also in relativistic systems.

Before we turn our attention to the full relativistic case it is useful to check if the particular example used by Andersson et al. [8] obeys the causality and absolute stability criteria derived above. First we note that, due to the presence of a velocity scale given by the speed of light, in relativity we cannot completely scale out the velocities. Thus the relativistic analysis of stability will in general contain an extra parameter compared to the Newtonian case. Here we shall take that parameter to be $c_{\mathrm{y}}$ which, without loss of generality, can be taken to be larger than $c_{\mathrm{x}}$. Using these parameters the absolute stability criterion (61) becomes just

$$
\frac{a^{2}}{b^{2}} \leq 1
$$

which is satisfied in the model discussed above. The causality condition (35) enters only indirectly as we have been able to re-scale in terms of $c_{\mathrm{y}}$ and thereby get dimensionless variables. We conclude that the Newtonian model of Andersson et al. is reasonable also from this perspective as long as $c_{\mathrm{y}}$ is not very close to the speed of light.

We now turn to the relativistic dispersion relation (59). Written as an equation for $\sigma_{\mathrm{x}}$ it constitutes a non-trivial quartic. If we use the same re-scalings as in (66), then (59) becomes

$$
\frac{x^{2}-b^{2}}{a^{2}}\left[\frac{(x-y)^{2}}{\gamma_{\mathrm{xy}}^{-2}\left(1-c_{\mathrm{y}}^{2} x^{2}\right)+c_{\mathrm{y}}^{2}(x-y)^{2}}-1\right]=1 .
$$



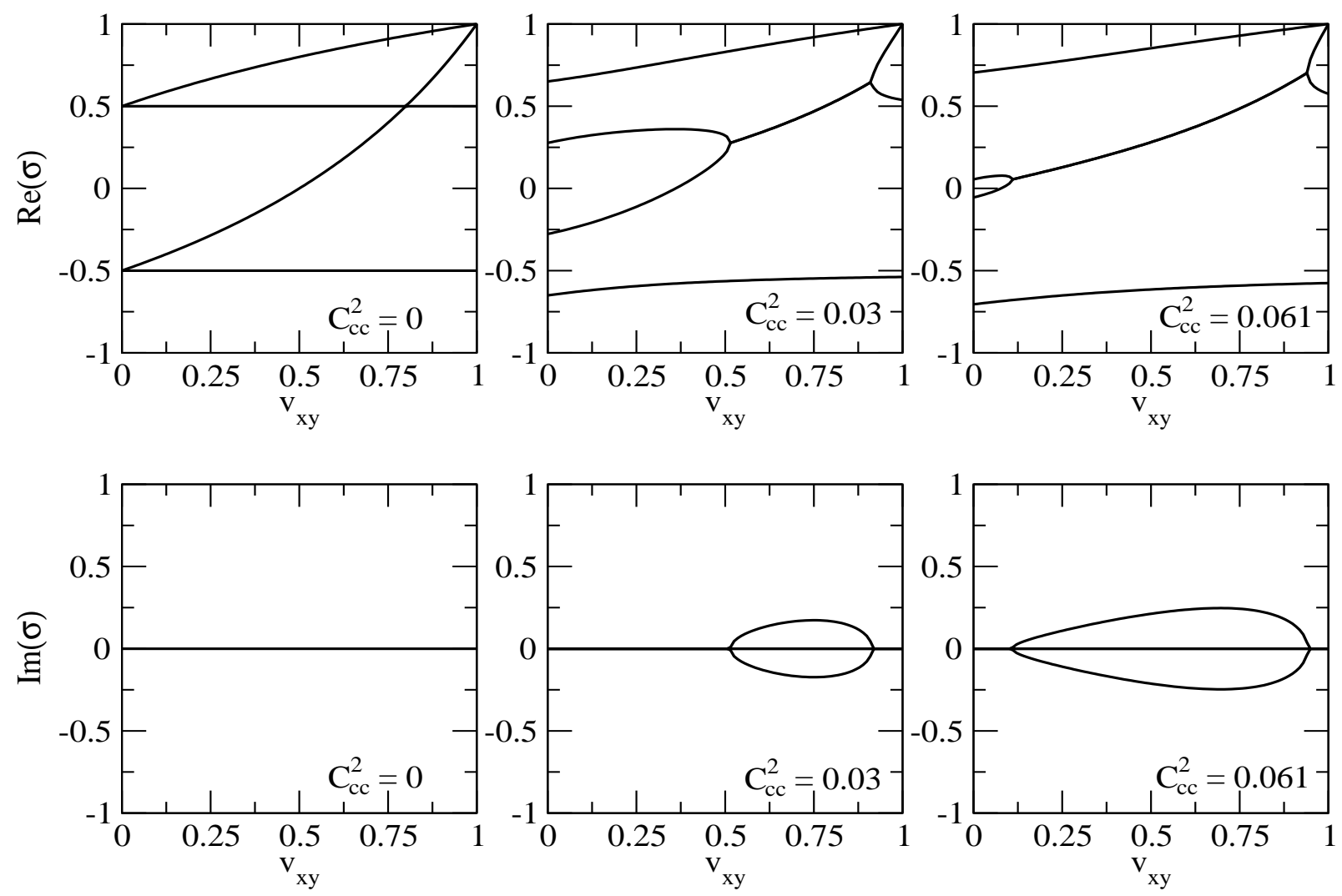

FIG. 1: Plots of the real (top) and imaginary (bottom) parts of the mode frequencies $\sigma_{\mathrm{x}}$ as functions of $v_{\mathrm{xy}}$, for $c_{y}=0.5$, $b^{2}=1.0$, and $\theta_{\mathrm{xy}}=0$. The merger of two frequencies, and subsequent non-zero imaginary values, signal the presence of a two-stream instability.

Some immediate insight is obtained by considering the ultra-relativistic limit for the background flow, where $v_{\mathrm{xy}} \rightarrow 1$ or $\gamma_{\mathrm{xy}}^{-2} \rightarrow 0$, and the limit where the wave vector becomes perpendicular to the background flow, i.e. $\theta_{\mathrm{xy}} \rightarrow \pi / 2$. In both limits the two-stream instability ceases to operate.

In the ultra-relativisitic limit, the wave-speed tends to

$$
\sigma_{\mathrm{x}} \rightarrow\left\{\begin{array}{l} 
\pm \sqrt{c_{\mathrm{x}}^{2}+\frac{\mathcal{c}_{c c}^{2}}{1-c_{\mathrm{y}}^{2}}} \\
\cos \theta_{\mathrm{xy}} \quad(\text { double root) }
\end{array} .\right.
$$

If the propagation is to remain causal, we must have $\mathcal{C}_{c c}^{2} \rightarrow 0$ as $c_{\mathrm{y}} \rightarrow 1$. Also there is no two-stream instability since $\mathcal{C}_{c c}^{2} \geq 0$. This might seem surprising, since a two-stream instability requires a "window" of background flows for modes to appear, say, left-moving in one frame but right-moving in the other. But as $v_{\mathrm{xy}} \rightarrow 1$ the relative flow is at its maximum, and yet the instability window is closed. In fact, this behaviour was seen already by Andersson et al. [8] in the Newtonian regime. From (66) we also see that $y \rightarrow 0$ as $\theta_{x y} \rightarrow \pi / 2$. This turns (69) into a quadratic for $x^{2}$, and one finds that the discriminant is positive for the range of values for $\mathcal{C}_{c c}^{2}$ that yield absolute stability and causality. Obviously, $y$ is the effective "window" of the background flow and it is completely closed for $\theta_{\mathrm{xy}}=\pi / 2$.

Although the general solution to (69) is readily availiable, it is quite complicated and offers very little additional insight. Instead of writing it down we will tackle the problem numerically. The parameter values are restricted to those that maintain absolute stability and causality. Figure 1 provides plots of the real and imaginary parts for the four solutions to (69) in the aligned case. The solutions for $\sigma_{\mathrm{x}}$ are taken to be functions of the relative flow parameter $y$ and the coupling $\mathcal{C}_{c c}^{2}$, with $c_{\mathrm{y}}=0.5, b^{2}=1$, and $\theta_{\mathrm{xy}}=0$. Non-zero values for $\operatorname{Im} \sigma_{\mathrm{x}}$ in the figures indicate the presence of an unstable mode. The appearance of unstable modes is reflected in the real parts wherever two frequencies merge. This behaviour is typical for this kind of dynamical instability. The results for misaligned flows, with $\theta_{\mathrm{xy}} \neq 0$ are very similar to those shown in Figure 1 As $\theta_{\mathrm{xy}}$ increases the $\left[y, \mathcal{C}_{c c}^{2}\right]$ region of instability moves towards higher relative velocities, eventually leading to regions that stretch essentially all the way to $v_{\mathrm{xy}}=1$. 


\section{Dispersion Relation: Aligned or Anti-aligned Flows}

Now that we have established the presence of the two-stream instability for arbitrary background flows, we will consider the more restricted case of aligned or anti-aligned background flows. This will simplify the dispersion relation so that analytical insight can be more easily acquired. It also reduces the parameter space, thus allowing a more focused numerical analysis.

By aligned or anti-aligned flow we mean that the wave propagation is aligned (or anti-aligned) with the relative velocity of the two fluids; specifically, $\theta_{\mathrm{xy}}=(0, \pi)$ so that

$$
\hat{k}_{a}^{\mathrm{x}}=\epsilon_{\mathrm{xy}} \hat{v}_{a}^{\mathrm{xy}} \quad, \quad \epsilon_{\mathrm{xy}} \equiv \cos \theta_{\mathrm{xy}}= \pm 1 .
$$

This leads naturally to the statement that the wave vector is a linear combination of the background flows:

$$
\begin{aligned}
k_{a} & =\frac{k_{\mathrm{x}}}{v_{\mathrm{xy}}}\left[\left(v_{\mathrm{xy}} \sigma_{\mathrm{x}}-\epsilon_{\mathrm{xy}}\right) u_{a}^{\mathrm{x}}+\epsilon_{\mathrm{xy}} \gamma_{\mathrm{xy}}^{-1} u_{a}^{\mathrm{y}}\right] \\
& =\frac{k_{\mathrm{y}}}{v_{\mathrm{xy}}}\left[\left(v_{\mathrm{xy}} \sigma_{\mathrm{y}}-\epsilon_{\mathrm{yx}}\right) u_{a}^{\mathrm{y}}+\epsilon_{\mathrm{yx}} \gamma_{\mathrm{xy}}^{-1} u_{a}^{\mathrm{x}}\right] .
\end{aligned}
$$

Equating coefficients in (72) leads to

$$
k_{a}=\frac{1}{\gamma_{\mathrm{xy}} v_{\mathrm{xy}}}\left(k_{\mathrm{y}} \epsilon_{\mathrm{yx}} u_{a}^{\mathrm{x}}+k_{\mathrm{x}} \epsilon_{\mathrm{xy}} u_{a}^{\mathrm{y}}\right) .
$$

Because of (73), our original four-dimensional linear algebra problem for $\left(u_{a}^{\mathrm{x}} A_{\mathrm{x}}^{a}, u_{a}^{\mathrm{x}} A_{\mathrm{y}}^{a}, u_{a}^{\mathrm{y}} A_{\mathrm{x}}^{a}, u_{a}^{\mathrm{y}} A_{\mathrm{y}}^{a}\right)^{\mathrm{T}}$ has been reduced to a two-dimensional one for $\left(u_{a}^{\mathrm{x}} A_{\mathrm{x}}^{a}, u_{a}^{\mathrm{y}} A_{\mathrm{y}}^{a}\right)^{\mathrm{T}}$.

\section{Dispersion Relation: Role of Entrainment}

Up to this point we have introduced three equation of state parameters $\left(c_{\mathrm{x}}, c_{\mathrm{y}}\right.$, and $\left.\mathcal{C}_{c c}\right)$ that are obtained as second derivatives of the master function. When entrainment is included in the model we see from (4) that we need two additional variables to describe the general case. Given that we have established the two-stream instability for general cross-constituent coupling and arbitrary background flow, the main reason for discussing the role of the entrainment is to highlight the basic feature that the instability can be triggered by a variety of interactions. We will simplify the entrainment case by assuming that the relative velocity $v_{\mathrm{xy}}^{a}$ is much smaller than the speed of light and that the flows are aligned (in the sense of the previous section). This does not mean, however, that the individual flows $u_{\mathrm{x}}^{a}$ have to be similarly restricted. Neither do the sound and wave speeds $c_{\mathrm{x}}$ and $\sigma_{\mathrm{x}}$ have to be small.

If we keep the relative flow to $\mathcal{O}\left(v_{\mathrm{xy}}^{2}\right)$, the master function can be approximated as [20, 21]

$$
\Lambda=\lambda_{0}\left(n_{\mathrm{x}}^{2}, n_{\mathrm{y}}^{2}\right)+\lambda_{1}\left(n_{\mathrm{x}}^{2}, n_{\mathrm{y}}^{2}\right)\left(n_{\mathrm{xy}}^{2}-\sqrt{n_{\mathrm{x}}^{2} n_{\mathrm{y}}^{2}}\right)
$$

which immediately implies

$$
\mathcal{B}^{\mathrm{x}}=-2\left[\frac{\partial \lambda_{0}}{\partial n_{\mathrm{x}}^{2}}+\frac{\partial \lambda_{1}}{\partial n_{\mathrm{x}}^{2}}\left(n_{\mathrm{xy}}^{2}-\sqrt{n_{\mathrm{x}}^{2} n_{\mathrm{y}}^{2}}\right)-\lambda_{1} \frac{n_{\mathrm{y}}}{2 n_{\mathrm{x}}}\right] \quad, \quad \mathcal{B}_{, \mathrm{xy}}^{\mathrm{x}}=-2 n_{\mathrm{x}} n_{\mathrm{y}} \frac{\partial \lambda_{1}}{\partial n_{\mathrm{x}}^{2}},
$$

and

$$
\mathcal{A}^{\mathrm{xy}}=-\lambda_{1} \quad, \quad \mathcal{A}_{, \mathrm{xy}}^{\mathrm{xy}}=0
$$

We shall make one further simplifying approximation, which is to take $\lambda_{1}$ to be constant so that

$$
\mathcal{B}_{, \mathrm{xy}}^{\mathrm{x}}=0
$$

This leaves us with the single entrainment parameter $\lambda_{1}$.

With these approximations we find

$$
\left[\begin{array}{cc}
\mathcal{B}^{\mathrm{x}}\left(\sigma_{\mathrm{x}}^{2}-c_{\mathrm{x}}^{2}\right) & -\mathcal{C}_{c c} \sqrt{\mathcal{B}^{\mathrm{x}} \mathcal{B}^{\mathrm{y}}}-\mathcal{A}^{\mathrm{xy}} \frac{\epsilon_{\mathrm{xy}} k_{\mathrm{x}}}{\epsilon_{\mathrm{yx}} k_{\mathrm{y}}}\left(\sigma_{\mathrm{x}}^{2}-1\right) \\
-\mathcal{C}_{c c} \sqrt{\mathcal{B}^{\mathrm{x}} \mathcal{B}^{\mathrm{y}}}-\mathcal{A}^{\mathrm{xy}} \frac{\epsilon_{\mathrm{yx}} k_{\mathrm{y}}}{\epsilon_{\mathrm{xy}} k_{\mathrm{x}}}\left(\sigma_{\mathrm{y}}^{2}-1\right) & \mathcal{B}^{\mathrm{y}}\left(\sigma_{\mathrm{y}}^{2}-c_{\mathrm{y}}^{2}\right)
\end{array}\right]\left[\begin{array}{c}
u_{a}^{\mathrm{x}} A_{\mathrm{x}}^{a} \\
u_{a}^{\mathrm{y}} A_{\mathrm{y}}^{a}
\end{array}\right]=\left[\begin{array}{l}
0 \\
0
\end{array}\right] .
$$


From (30) and (42) we have

$$
\frac{k_{\mathrm{x}}}{k_{\mathrm{y}}}=\sqrt{\frac{1-\sigma_{\mathrm{y}}^{2}}{1-\sigma_{\mathrm{x}}^{2}}}
$$

and thus the dispersion relation becomes

$$
0=\left(\sigma_{\mathrm{x}}^{2}-c_{\mathrm{x}}^{2}\right)\left(\sigma_{\mathrm{y}}^{2}-c_{\mathrm{y}}^{2}\right)-\left[\mathcal{C}_{c c}+\mathcal{C}_{e n t} \sqrt{\left(1-\sigma_{\mathrm{x}}^{2}\right)\left(1-\sigma_{\mathrm{y}}^{2}\right)}\right]^{2},
$$

where

$$
\mathcal{C}_{\text {ent }}=\epsilon_{\mathrm{xy}} \epsilon_{\mathrm{yx}} \frac{\mathcal{A}^{\mathrm{xy}}}{\sqrt{\mathcal{B}^{\mathrm{x}} \mathcal{B}^{\mathrm{y}}}},
$$

and because the inverse of (7) must exist, $\left|\mathcal{C}_{\text {ent }}\right| \neq 1$. Note that, since $\sigma_{\mathrm{y}}^{2} \rightarrow 1$ as $v_{\mathrm{xy}} \rightarrow 1$, $\mathcal{C}_{\text {ent }}$ does not affect the ultra-relativistic limit. Hence, equation (70) $\left[\right.$ with $\left.\cos \left(\theta_{\mathrm{xy}}\right)= \pm 1\right]$ is still valid in this case.

We stress that, unlike the simpler cases, 800 does not hold for arbitrary propagation direction with respect to the relative velocity. This is important qualitatively and quantitatively, since it mirrors the fact that entrainment enters the master function in a fundamentally different way: At first-order in the relative velocity squared. In the dispersion relation, however, entrainment contributes even in the limit of zero relative velocity, because there are still two sets of interacting sound waves. In fact, we will now follow the earlier analysis of causality and absolute stability by taking this limit.

From (44) we see $\sigma_{\mathrm{x}}^{2}=\sigma_{\mathrm{y}}^{2}$ so that

$$
\left(\sigma_{\mathrm{x}}^{2}-c_{\mathrm{x}}^{2}\right)\left(\sigma_{\mathrm{x}}^{2}-c_{\mathrm{y}}^{2}\right)-\left[\mathcal{C}_{c c}+\mathcal{C}_{\text {ent }}\left(1-\sigma_{\mathrm{x}}^{2}\right)\right]^{2}=0
$$

It is particularly instructive to consider the entrainment alone, i.e. set $\mathcal{C}_{c c}=0$. The corresponding dispersion relation is a quadratic in $\sigma_{\mathrm{x}}^{2}$, and has the solutions

$$
\sigma_{\mathrm{x}}^{2}=\frac{c_{\mathrm{x}}^{2}+c_{\mathrm{y}}^{2}-2 \mathcal{C}_{e n t}^{2} \pm\left[\left(c_{\mathrm{x}}^{2}-c_{\mathrm{y}}^{2}\right)^{2}+4 \mathcal{C}_{e n t}^{2}\left(1-c_{\mathrm{x}}^{2}\right)\left(1-c_{\mathrm{y}}^{2}\right)\right]^{1 / 2}}{2\left(1-\mathcal{C}_{\text {ent }}^{2}\right)}
$$

For $c_{\mathrm{x}, \mathrm{y}}^{2} \leq 1$, the discriminant is obviously positive and hence the $\sigma_{\mathrm{x}}^{2}$ are real. In order to analyze absolute stability and causality we need to consider the ranges $0 \leq \mathcal{C}_{\text {ent }}^{2}<1$ and $1<\mathcal{C}_{\text {ent }}^{2}$ separately. We will look at $\mathcal{C}_{\text {ent }}^{2}>1$ first.

The first step is to re-write (83) so that the denominator is positive:

$$
\sigma_{\mathrm{x}}^{2}=\frac{2 \mathcal{C}_{e n t}^{2}-c_{\mathrm{x}}^{2}-c_{\mathrm{y}}^{2} \pm\left[\left(c_{\mathrm{x}}^{2}-c_{\mathrm{y}}^{2}\right)^{2}+4 \mathcal{C}_{e n t}^{2}\left(1-c_{\mathrm{x}}^{2}\right)\left(1-c_{\mathrm{y}}^{2}\right)\right]^{1 / 2}}{2\left(\mathcal{C}_{e n t}^{2}-1\right)}
$$

Since we are imposing $c_{\mathrm{xy}}^{2} \leq 1$, and $\mathcal{C}_{\text {ent }}^{2}>1$, the terms outside the square root in the numerator are positive. Therefore, the "+" solution is absolutely stable. But, we can also show that it cannot be causal. As for the "-" solution, we can easily show that it is absolutely stable only if $\mathcal{C}_{\text {ent }}^{2}<1$, which cannot be satisfied. Hence, $\mathcal{C}_{\text {ent }}^{2}>1$ does not lead to both absolute stability and causality, and is therefore ruled out.

The range $0 \leq \mathcal{C}_{e n t}^{2}<1$ is a different story, because the terms outside the radical in the numerator of (83) are not of any definite sign. This affects the absolute stability analysis more than the determination of causality. In fact, the causality analysis is sufficiently straightforward that we will simply state that this requirement is satisfied for this range of $\mathcal{C}_{\text {ent }}^{2}$. In order to assess absolute stability, it is useful to introduce

$$
\tau=\left[\left(c_{\mathrm{x}}^{2}-c_{\mathrm{y}}^{2}\right)^{2}+4 \mathcal{C}_{e n t}^{2}\left(1-c_{\mathrm{x}}^{2}\right)\left(1-c_{\mathrm{y}}^{2}\right)\right]^{1 / 2}
$$

This allows the numerator of (83) to be rewritten in such a way that the absolute stability condition becomes

$$
\tau^{2}-\left[ \pm 2\left(1-c_{\mathrm{x}}^{2}\right)\left(1-c_{\mathrm{y}}^{2}\right)\right]+\left[c_{\mathrm{x}}^{2}\left(1-c_{\mathrm{y}}^{2}\right)+c_{\mathrm{y}}^{2}\left(1-c_{\mathrm{x}}^{2}\right)\right]\left[2-\left(c_{\mathrm{x}}^{2}+c_{\mathrm{y}}^{2}\right)\right] \leq 0
$$

where the " \pm " corresponds to that of (83). The final step is to factorize (86) and thereby obtain

$$
\left\{\tau \pm\left[2-\left(c_{\mathrm{x}}^{2}+c_{\mathrm{y}}^{2}\right)\right]\right\}\left\{\tau \mp\left[c_{\mathrm{x}}^{2}\left(1-c_{\mathrm{y}}^{2}\right)+c_{\mathrm{y}}^{2}\left(1-c_{\mathrm{x}}^{2}\right)\right]\right\} \leq 0
$$



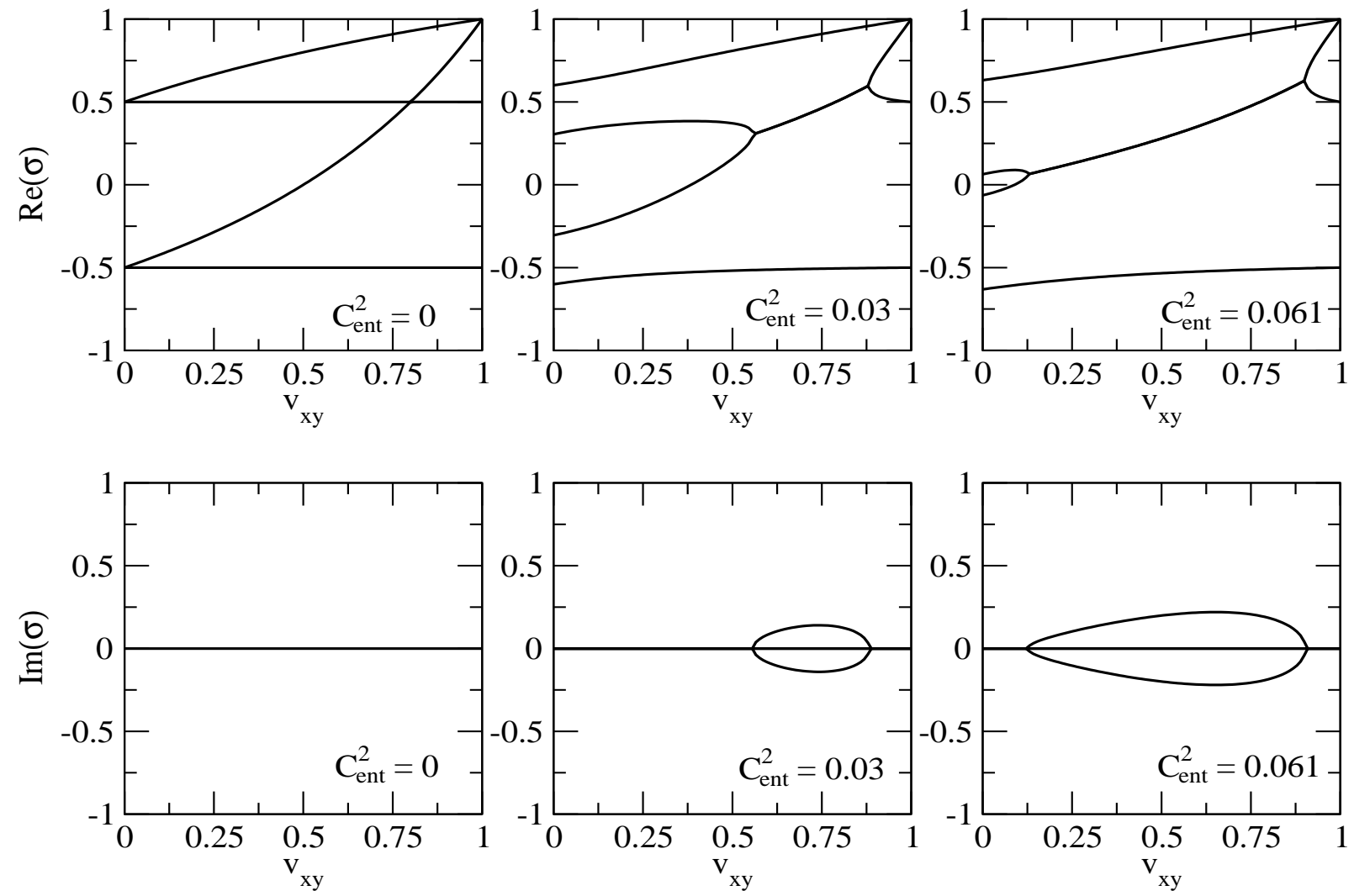

FIG. 2: Plots of the real and imaginary parts of the mode frequencies $\sigma_{\mathrm{x}}$ as functions of $y$ and $\mathcal{C}_{\text {ent }}^{2}$, for $c_{y}=0.5$, and $b^{2}=1.0$. The lines of intersection and subsequent merger of two frequencies signal the presence of a two-stream instability.

where if the "+" is taken from the first factor then the "-" must be taken in the second, and vice versa. In either case, the factor that has the "+" is positive definite, and so the other factor must be less than zero. When the first factor takes the "-" the inequality leads to $\mathcal{C}_{\text {ent }}^{2} \leq 1$. The other choice leads to the more restrictive condition of $\mathcal{C}_{e n t}^{2} \leq c_{\mathrm{x}}^{2} c_{\mathrm{y}}^{2}$.

To summarize, we have shown that when $\mathcal{C}_{\text {ent }}^{2}>1$, there is either no absolute stability or causality, which makes this range unphysical. Meanwhile, for $0 \leq \mathcal{C}_{\text {ent }}^{2} \leq c_{\mathrm{x}}^{2} c_{\mathrm{y}}^{2}$ the system is causal and absolutely stable. In terms of our earlier definitions, this translates into

$$
\left(\mathcal{A}^{\mathrm{xy}}\right)^{2} \leq \mathcal{B}^{\mathrm{x}} \mathcal{B}^{\mathrm{y}}\left(\frac{\partial \log \mathcal{B}^{\mathrm{x}}}{\partial \log n_{\mathrm{x}}}+1\right)\left(\frac{\partial \log \mathcal{B}^{\mathrm{y}}}{\partial \log n_{\mathrm{y}}}+1\right)
$$

as a constraint on the master function, when the relative speed between the two fluids is sufficiently small.

Finally, we turn to a numerical/graphical analysis for exposing the two-stream instability due to entrainment coupling. As in the cross-constituent coupling case, we use the re-scalings of (66), except that $\mathcal{C}_{\text {ent }}^{2}$ replaces $\mathcal{C}_{c c}^{2}$ in $a^{2}$. Equation (80) becomes

$$
\frac{x^{2}-b^{2}}{a^{2}}\left[\frac{(x-y)^{2}}{\left(c_{\mathrm{y}}^{2} y x-1\right)^{2}}-1\right]=\left(c_{\mathrm{y}}^{2} x^{2}-1\right)\left[\frac{c_{\mathrm{y}}^{2}(x-y)^{2}}{\left(c_{\mathrm{y}}^{2} y x-1\right)^{2}}-1\right] .
$$

The parameter values are restricted to those that maintain absolute stability and causality. Fig. 2 plots the real and imaginary parts of the four solutions to (89). As before, the solutions for $\sigma_{\mathrm{x}}$ are taken to be functions of the relative flow parameter $y$ and the coupling $\mathcal{C}_{\text {ent }}^{2}$, with $c_{\mathrm{y}}=0.5$ and $b^{2}=1.0$. Fig. 2 is not so dissimilar from what we find for cross-constituent coupling, thus highlighting that the instability is not sensitive to the type of coupling between the two fluids. 


\section{CLOSING REMARKS}

There are several examples of relativistic systems that require multi-fluid dynamics for qualitative understanding and quantitative accuracy. In such systems different interpenetrating fluid components (eg. particles and entropy in heat conducting situations or a superfluid condensate and finite temperature excitations) can flow with distinct velocities.

We have examined plane-wave propagation for a generic two-fluid system. By imposing the constraints of absolute stability (necessary in order for the components not to separate already in the absence of flow) and causality we have established limits on the equation of state (as represented by the master function $\Lambda$ ). In particular, we place constraints on the free sound speeds, the cross-constituent coupling and the entrainment. Some of the obtained results are more or less trivial extensions of the single fluid result, but others are unique to the two-fluid problem. The condition (88) on the entrainment is a new result, and as such serves as a new condition on, say, the kind of $\sigma-\omega$ model used by Comer and Joynt [15] to model entrainment in the outer cores of neutron stars.

We have demonstrated (for the first time) the existence of a relativistic two-stream instability. This is a generic phenomenon, that does not require particular fine-tuning to be triggered, nor is it limited to any specific physical system. The only requirement is that there is a relative (background) flow and some type of coupling between the fluids. While it is true that a single fluid can have an analogous instability, it is only active at an interface where there is shearing motion. Our analysis assumes that the two fluids are interpenetrating.

In order to exhibit the generic nature of the two-stream instability, we have kept the analysis rather abstract. On the one hand, this means that it should be relatively straightforward to apply our results to particular physical systems. On the other hand, it means that we have not yet discussed the relevance of the instability for any particular system. A more detailed consideration of multi-fluid problems in relativity is required in order to establish whether this mechanism operates in nature. There are already exciting results that hint at this class of instabilities being associated with pulsar glitches [8, 9]. In addition to exploring possible situations where these instabilities may operate, it would be very interesting to probe the nonlinear development of the unstable waves. So far, all studies have been at the linear perturbation level. The results establish the presence of the instability, but they do not shed any light on what happens once the unstable oscillation reaches nonlinear amplitudes. Detailed studies of this problem are essential if we are to understand the actual dynamical role of this instability.

\section{Acknowledgments}

NA acknowledges support from STFC via grant number PP/E001025/1. CLM is supported by CONACyT. GLC acknowledges partial support from NSF via grant number PHYS-0855558.

[1] D. T. Farley, Phys. Rev. Lett. 10, 279 (1963).

[2] O. Buneman, Phys. Rev. 115, 503 (1959).

[3] F. F. Chen, Introduction to plasma physics and controlled fusion (Plenum, New York, 1984).

[4] R. V. E. Lovelace, K. P. Jore, and M. P. Haynes, Astrophys. J. 475, 83 (1997).

[5] S. Chandrasekhar, Phys. Rev. Lett. 24, 611 (1970).

[6] J. L. Friedman and B. F. Schutz, Ap. J. 222, 281 (1978).

[7] N. Andersson, G. L. Comer, and R. Prix, Phys. Rev. Lett. 90, 091101 (2003).

[8] N. Andersson, G. L. Comer, and R. Prix, Mon. Not. R. Astro. Soc. 354, 101 (2004).

[9] K. Glampedakis and N. Andersson, Physical Review Letters 102, 141101 (2009), 0806.3664.

[10] C. W. Misner, K. S. Thorne, and J. A. Wheeler, Gravitation (San Francisco, US: W.H. Freeman and Co., 1973).

[11] B. Carter, in Relativistic Fluid Dynamics (Noto, 1987), edited by A. Anile and M. Choquet-Bruhat (Springer-Verlag, Heidelberg, Germany, 1989), vol. 1385 of Lecture Notes in Mathematics, pp. 1-64.

[12] N. Andersson and G. L. Comer, Living Reviews in Relativity 10, 1 (2007).

[13] A. F. Andreev and E. P. Bashkin, Sov. Phys. JETP 42, 164 (1975).

[14] M. Borumand, R. Joynt, and W. Kluzniak, Phys. Rev. C 54, 2745 (1996).

[15] G. L. Comer and R. Joynt, Phys. Rev. D 68, 023002 (2003).

[16] N. Chamel and P. Haensel, Phys. Rev. C 73, 045802 (2006), arXiv:nucl-th/0603018.

[17] N. Andersson and G. L. Comer, ArXiv e-prints (2008), 0811.1660.

[18] B. Carter and L. Samuelsson, Class. Quant. Grav. 23, 5367 (2006).

[19] S. Weinberg, Gravitation and Cosmology: Principles and Applications of the General Theory of Relativity (New York, US: Wiley, 1972).

[20] N. Andersson, G. L. Comer, and D. Langlois, Phys. Rev. D 66, 104002 (2002). 
[21] N. Andersson and G. Comer, Mon. Not. R. Astro. Soc. 328, 1129 (2001).

[22] B. Carter and I. Khalatnikov, Rev. Math. Phys. 6, 277 (1994).

[23] It is worth making the following remark on the notation. Throughout the paper we only consider two fluids. They are generally labelled by $\mathrm{x}$ and $\mathrm{y}$. However, in order to be economic in the presentation we often treat the constituent index as abstract, meaning that it can be either $\mathrm{x}$ or $\mathrm{y}$. That is, an equation written down explicitly for fluid $\mathrm{x}$ takes exactly the same form for the other fluid once the index $\mathrm{x}$ is replaced by $\mathrm{y}$ (and vice versa). We are aware that this convention may be confusing at first, but it makes sense. Especially if one wants to account for additional fluid components. Most of our equations still remain valid in that case, although in each equation for fluid $\mathrm{x}$ one has to sum over all the other fluids (i.e. the sum runs over $\mathrm{y} \neq \mathrm{x}$ ).

[24] In fact, the celebrated Landau model for superfluid Helium mixes a momentum with a flux, see for example, the discussion in 22$]$. 